\title{
Metabolic scavenging by cancer cells: when the going gets tough, the tough keep eating
}

\author{
Evdokia Michalopoulou ${ }^{1,2}$, Vinay Bulusu ${ }^{1,2}$ and Jurre J Kamphorst ${ }^{\star, 1,2}$ \\ ${ }^{1}$ Cancer Metabolism Research Unit, Cancer Research UK Beatson Institute, Garscube Estate, Switchback Road, Glasgow G61 1BD, \\ UK and ${ }^{2}$ Institute of Cancer Sciences, University of Glasgow, Garscube Estate, Switchback Road, Glasgow G61 10H, UK
}

\begin{abstract}
Cancer is fundamentally a disease of uncontrolled cell proliferation. Tumour metabolism has emerged as an exciting new discipline studying how cancer cells obtain the necessary energy and cellular 'building blocks' to sustain growth. Glucose and glutamine have long been regarded as the key nutrients fuelling tumour growth. However, the inhospitable tumour microenvironment of certain cancers, like pancreatic cancer, causes the supply of these nutrients to be chronically insufficient for the demands of proliferating cancer cells. Recent work has shown that cancer cells are able to overcome this nutrient insufficiency by scavenging alternative substrates, particularly proteins and lipids. Here, we review recent work identifying the endocytic process of macropinocytosis and subsequent lysosomal processing as an important substrate-acquisition route. In addition, we discuss the impact of hypoxia on fatty acid metabolism and the relevance of exogenous lipids for supporting tumour growth as well as the routes by which tumour cells can access these lipids. Together, these cancer-specific scavenging pathways provide a promising opportunity for therapeutic intervention.
\end{abstract}

Unremitting cell proliferation, the true hallmark of cancer, is metabolically demanding. It requires energy, reducing power, and cellular 'building blocks' in the form of amino acids for proteins, fatty acids for lipids and nucleotides for DNA and RNA. Despite the inherently intimate link between proliferation and metabolism, which was already demonstrated early on by Warburg et al (1927, 1956), the field of cancer metabolism has only recently risen to prominence. An important instigator was the realisation that the observed metabolic reprogramming in cancer cells is directly triggered by oncogenes (Deberardinis et al, 2008). In fact, the oncogene-induced autonomous acquisition of nutrients has been proposed to be a critical step in cellular transformation (Pavlova and Thompson, 2016). One of the first signalling cascades to be studied in the context of cancer metabolism is the $\mathrm{PI}(3) \mathrm{K} / \mathrm{Akt} / \mathrm{mTOR}$ pathway, which is often constitutively activated in cancer (Elstrom et al, 2004; Engelman et al, 2006). In keeping with its physiological role as the insulin effector pathway, activation of this pathway results in enhanced glucose uptake and increased de novo synthesis of cellular building blocks (Figure 1A).

Recent work has emphasised that the stressful conditions of the tumour microenvironment, with parts of the tumour periodically experiencing limited availability of primary nutrients and oxygen, also affect the metabolism of cancer cells. Limited oxygen availability affects central carbon metabolism, and more recently has been shown to compromise fatty acid synthesis (Kamphorst et al, 2013; Young et al, 2013). The strong influence of the microenvironment is epitomised in pancreatic cancer. Pancreatic ductal adenocarcinomas (PDAC) are characterised by high interstitial pressures, leading to blood vessel collapse and diminished nutrient and oxygen delivery (Provenzano et al, 2012; Chauhan et al, 2014; Delgiorno et al, 2014). Despite this, $\mathrm{PDAC}$ is one of the most aggressive cancers. Research into the metabolism of PDAC cells revealed that they scavenge extracellular proteins by hijacking an ancient feeding mechanism called macropinocytosis to support metabolism and proliferation (Figure 1B).

Scavenging of alternative nutrients, particularly macromolecules, has emerged as an alternative to de novo synthesis to support proliferation of cancer cells, and has recently been included as one of the hallmarks of cancer metabolism (Pavlova and Thompson, 2016). Here, we review recent studies on metabolic scavenging by cancer cells, with a particular emphasis on macropinocytosis and lipid scavenging. We also discuss remaining questions and highlight the exciting therapeutic potential of targeting these novel scavenging pathways.

*Correspondence: Dr JJ Kamphorst; E-mail: Jurre.Kamphorst@glasgow.ac.uk

Received 27 May 2016; revised 14 July 2016; accepted 19 July 2016; published online 18 August 2016 


\section{GOING BIG_EATING PROTEINS TO SURVIVE AND GROW}

The idea that cancer cells degrade macromolecules to support metabolism is not new. In fact, a well-established mechanism exists by which cells degrade intracellular material: autophagy. During autophagy, cytoplasmic macromolecules, protein aggregates and/or organelles are sequestered into double-membraned vesicles known as autophagosomes. These fuse with lysosomes, allowing lysosomal enzymes (proteases, lipases, nucleases and glycosidases) to degrade the lysosomal content. Autophagy functions as the 'waste-disposal' system in cells, clearing damaged macromolecules and organelles that would otherwise become harmful. As a consequence of lysosomal hydrolysis metabolites such as amino acids, fatty acids, nucleosides and sugars are released from the lysosomes, ready to be re-used for energy production or as cellular building blocks (Rabinowitz and White, 2010).

It has been proposed that in an established tumour autophagy promotes tumour cell survival and resistance against therapy. In particular, multiple reports have highlighted the importance of autophagy in the context of oncogenic Ras-driven tumours. Cells expressing oncogenic Ras were found to have elevated basal autophagy (Karsli-Uzunbas et al, 2014). In keeping with its metabolic function, disrupting the autophagy machinery in these cells led to reduced TCA cycle activity and a depletion of energy, resulting in reduced viability during starvation conditions in vitro and reduced tumourigenesis in vivo. It thus appears that autophagy is particularly important for maintaining mitochondrial activity, both by ensuring a healthy mitochondrial pool and by replenishing the TCA cycle with autophagic breakdown products, including amino acids like glutamine (Strohecker and White, 2014). In a separate study, an elevation in basal autophagy was observed in PDAC, a tumour type with a near $100 \%$ occurrence of mutant KRAS (Yang et al, 2011). Inhibition of autophagy attenuated proliferation of PDAC cells, and in vivo PDAC tumour progression was similarly severely affected. This was due to increased ROS production and decreased mitochondrial oxidative phosphorylation, again highlighting the importance of autophagy in metabolism. Further evidence for increased dependence on autophagy and lysosomal processing in PDAC was provided by a study showing their aberrant regulation (Perera et al, 2015). Specifically, the MiT/ TFE transcription factors were found to exhibit increased nuclear residence through shuttling by IPO8/IPO7, which led to the upregulation of autophagy- and lysosome-promoting genes. Disruption of these transcription factors resulted in reduced cellular amino acid pools. From these studies it is apparent that Ras-driven cancers, particularly PDAC, hijack autophagy to maintain metabolic homoeostasis.

An endocytic mechanism resembling autophagy is macropinocytosis. It occurs at the cell surface, where membrane ruffling leads to the engulfment of extracellular fluid and formation of cytoplasmic vesicles called macropinosomes (Figure 1B). A first hint at its potential importance in cancer was provided by the finding that macropinocytosis is induced by Ras. Although this observation was made some 30 years ago, only recently was it discovered that macropinocytosis functions to take in macromolecules to support metabolism (Commisso et al, 2013). In this study it was shown that macropinocytosis-mediated uptake of extracellular protein (albumin) and subsequent hydrolysis provides amino acids to support central carbon metabolism. Macropinocytosis digests extracellular material, and because of this it accrues new biomass and hence facilitates growth and proliferation, and not just survival. Recent work confirmed that macropinocytosis occurs in human PDAC, and that it enables continued growth of PDAC cells in the absence of free essential amino acids (Kamphorst et al, 2015). Together, these reports argue that macropinocytosis is an important mode of nutrient acquisition in PDAC.
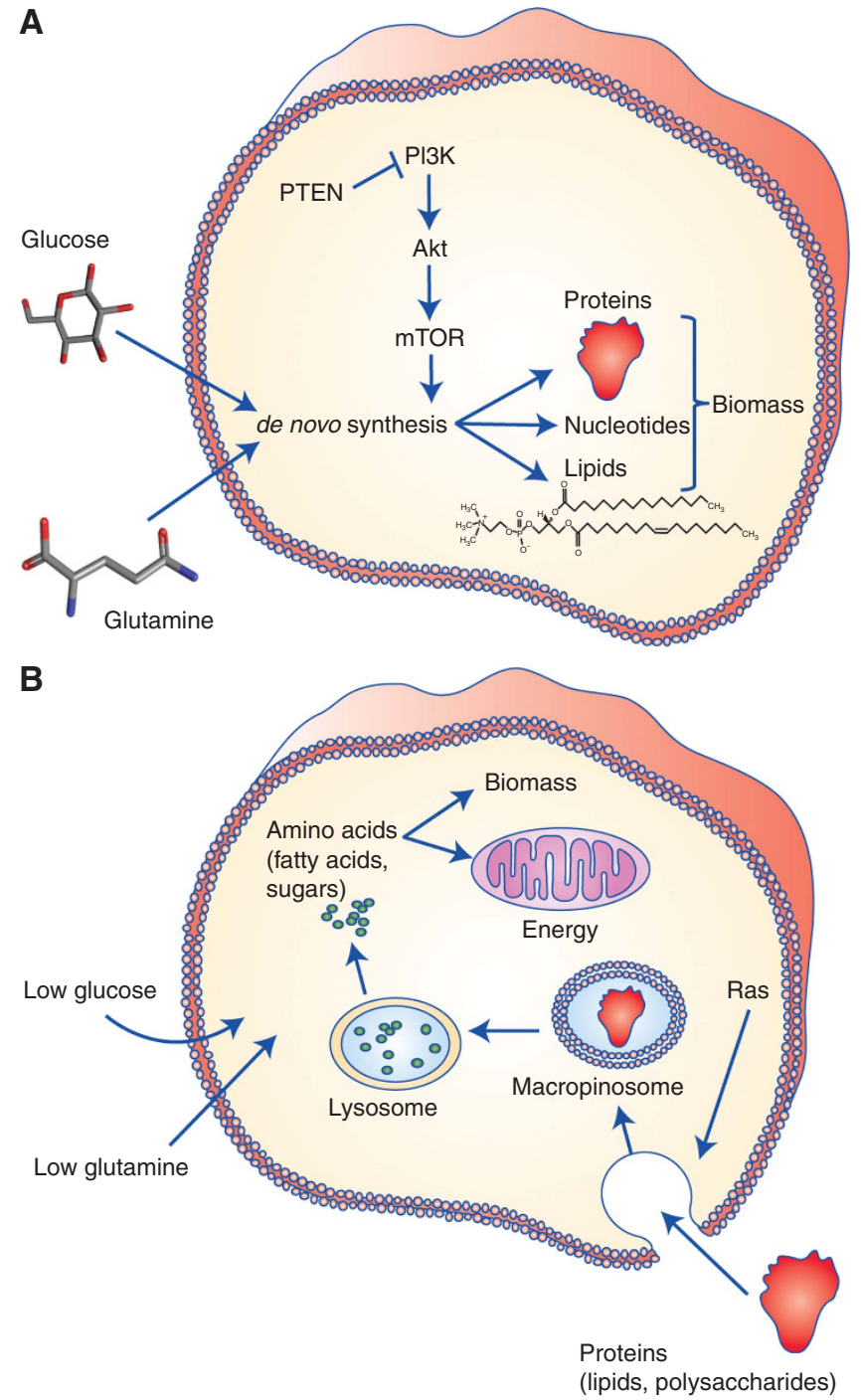

Figure 1. Cancer cells can use two modes of metabolism to acquire cellular 'building blocks' for growth. (A) An established mode, most extensively studied in the context of PI(3)K-Akt-mTOR signalling, involves de novo synthesis of cellular building blocks (amino acids, nucleotides, fatty acids) from glucose and free amino acids, particularly glutamine, for the construction of new daughter cells. (B) More recently, an alternative mode was discovered that allows certain cancer types (PDAC in particular) to maintain survival and proliferation in nutrient poor conditions, by scavenging alternative nutrients, particularly protein and lipid macromolecules. Extracellular proteins can be consumed by an endocytic process called macropinocytosis. Lipidscavenging pathways are depicted in detail in Figure 2.

The regulation of autophagy is relatively well-described. macropinocytosis, on the other hand, has until now received much less attention, and much remains to be learned about its regulation, especially in mammalian systems. A recent advance was made by the establishment of a link between mTORC1 and extracellular protein scavenging (Palm et al, 2015). In this study, it was corroborated that cells with mutant KRAS were able to proliferate in medium lacking leucine when albumin was provided. Cells expressing activated Akt1 (myr-Akt1) on the other hand, could not. Surprisingly, the authors found that while under nutrient-replete conditions mTORC1 inhibition attenuated growth of cells with mutant KRAS, during free amino acid-depleted conditions it actually led to increased lysosomal degradation of 
extracellular protein and increased proliferation. The organism most extensively used for macropinocytosis research is the soildwelling amoebae Dictyostelium discoideum. Dictyostelium uses macropinocytosis to feed on bacteria. Over time, lab strains with heightened macropinocytosis have evolved, allowing them to grow on liquid medium. Interestingly, these lab strains commonly have mutations in a Ras-GAP (Neurofibromin 1) leading to constitutive activation of Ras (Bloomfield et al, 2015). Various studies in Dictyostelium have identified major regulators of macropinocytosis such as the small GTPases Rac1 and Cdc42 (Dumontier et al, 2000). Furthermore, it has been shown that the Arp2/3 complex along with phosphoinositide 3-kinase are required for the formation of macropinosomes ( Hoeller et al, 2013). The requirement for PIP3 lipids in macropinosome formation seemingly contradicts the finding that the PI(3)K/Akt/mTOR pathway (also depending on PIP3) is a negative regulator of extracellular protein scavenging. However, the $\mathrm{PI}(3) \mathrm{Ks}$ in both situations are likely to be different isoforms and/or they may be exposed to different stimuli simply because of their location (plasma membrane surface $v s$ macropinosome).

The regulatory complexity of macropinocytosis is likely to be of similar scale as that of autophagy. Owing to the renewed interest, we expect that important aspects of this process will soon be elucidated. Beyond the regulation of macropinocytosis, however, many questions remain. Is PDAC the only type of cancer to use macropinocytosis as a feeding mechanism? What macromolecules do tumour cells scavenge in vivo? Can they consume extracellular matrix, and would that enable increased migration? Does macropinocytosis confer resistance to therapies that inhibit biosynthesis or angiogenesis? Arguably the most important question is, however, does macropinocytosis itself provide a therapeutic opportunity? Although a better understanding of macropinocytosis will help answering this question, results so far are promising. Because macropinosomes end up fusing with lysosomes, inhibiting lysosomal function can inhibit extracellular protein scavenging. The chloroquine derivative hydroxychloroquine (HCQ) inhibits the acidification of lysosomes and shows promising results when combined with gemcitabine (Boone et al, 2015). At the moment, multiple clinical trials with HCQ are ongoing. Other lysosomal inhibitors are currently being developed and it would be extremely interesting to study their effect in PDAC.

A radically different approach is to exploit PDAC's taste for protein to selectively smuggle chemotherapeutics into cancer cells to reach high therapeutic doses (the 'Trojan horse' approach). Tantalising results supporting this idea were obtained in a clinical study with the protein-drug conjugate albumin-paclitaxel (nab-paclitaxel). In this study with 861 patients, the combination of nab-paclitaxel with gemcitabine significantly increased the overall survival compared with gemcitabine treatment alone (Goldstein et al, 2015). On the basis of these encouraging results, further investigation into exploiting macropinocytosis for therapeutic purposes is certainly warranted.

\section{GREASING THE CANCER MACHINE-SCAVENGING} ACETATE, FATTY ACIDS AND LIPIDS

Fatty acids are a sine qua non for cellular proliferation. Whereas the essential poly-unsaturated fatty acids (PUFA) can only be obtained by uptake from dietary sources, cells have the option to either build non-essential fatty acids from scratch or to take them up. A considerable body of research indicates that, in contrast to most other tissue types, many cancers engage in de novo fatty acid synthesis. Especially cancer cells with a constitutively active PI(3)K-Akt-mTOR axis, most commonly through loss of PTEN or activating mutations in $\mathrm{PI}(3) \mathrm{K}$, are reliant on lipogenesis
(Porstmann et al, 2008). Signalling by mTORC1 activates the sterol-regulatory element-binding protein family of transcription factors (Porstmann et al, 2008), inducing the expression of the lipogenic genes such as acetyl-CoA carboxylase (ACACA), fatty acid synthase (FASN) and stearoyl-CoA desaturase (SCD), to facilitate increased fatty acid biosynthesis. Further evidence for an important role for lipogenesis in cancer has been found in the upregulation of FASN in a variety of tumours. It is, however, important to note that enzyme expression per se does not necessarily correlate with the actual lipogenic flux. Cells could upregulate FASN in an effort to maintain fatty acid biosynthesis during nutrient limitation.

The precursor for fatty acid synthesis is acetyl-CoA (AcCoA), whose acetyl-unit in normoxic cells is derived mostly from glucose. Hypoxia, a common occurrence in solid tumours, is known to affect central carbon metabolism; in a HIF1-mediated suppression of pyruvate dehydrogenase activity most glucose is shunted towards lactate, instead of entering the TCA cycle (Kim et al, 2006; Papandreou et al, 2006). This affects AcCoA production from glucose and how this in turn affects fatty acid synthesis has been actively researched in recent years. A first insight into this came from multiple publications showing that in (pseudo)hypoxic cells fatty acids had strongly increased ${ }^{13} \mathrm{C}$-carbon labelling from ${ }^{13} \mathrm{C}$-glutamine compared with normoxic cells (Wise et al, 2011; Metallo et al, 2012; Mullen et al, 2012). Notably, this increased labelling occurred through the reductive production of the AcCoA precursor citrate from glutamine-derived $\alpha$-ketoglutarate, rather than through oxidative metabolism. Later work argued that the increased fatty acid labelling from ${ }^{13} \mathrm{C}$-glutamine in hypoxia can at least partly be explained by exchange of intermediates rather than a net synthesis flux (Fan et al, 2013). Many reactions in metabolism are reversible, as is the reaction between $\alpha$-ketoglutarate and citrate. When using ${ }^{13} \mathrm{C}$-glutamine, the resulting labelled $\alpha$ ketoglutarate can mix with citrate, causing it also to become labelled, even when there is no net synthesis of citrate from $\alpha$ ketoglutarate. As citrate itself is the precursor for AcCoA which is used for fatty acid synthesis, it is possible that labelling of fatty acids occurs from ${ }^{13} \mathrm{C}$-glutamine without a net carbon contribution. Therefore, to what degree carbon from glutamine actually contributes to fatty acid synthesis in hypoxic cells remains debated. As stated, reversibility of reactions is a common phenomenon in metabolism, and this should always be considered in the interpretation of stable-isotope-tracing experiments.

More recently another substrate that could potentially sustain fatty acid synthesis in hypoxic cancer cells was identified: acetate. Specifically, it was found that the enzyme generating AcCoA from acetate, acetyl-CoA synthetase 2 (ACSS2), is frequently amplified in a subset of cancers and is upregulated in cells exposed to hypoxic and low serum conditions (Schug et al, 2015). Consequently, labelling of lipogenic AcCoA and hence fatty acids from ${ }^{13} \mathrm{C}$-acetate was found to be increased in these conditions (Kamphorst et al, 2014; Schug et al, 2015). Further work showed a significant reduction in tumour growth upon knockdown of ACSS2 in a breast cancer xenograft model (Schug et al, 2015), and a significant reduction in tumour burden was found following ACSS2 knockout in a genetically engineered mouse model of hepatocellular carcinoma (Comerford et al, 2014). These observations clearly demonstrate the importance of ACSS2 in maintaining tumour growth of certain cancer types. However, how exactly this occurs, is not yet fully understood. Although a likely explanation is that ACSS2 captures acetate as an alternative carbon source to support biomass production when AcCoA production from glucose is limited, formal proof for this is lacking. Direct assessment of acetate uptake rates by cells is required to determine if net uptake occurs in hypoxic cells and if it is higher than in normoxic cells. This would help establish whether increased labelling in hypoxic cells from ${ }^{13} \mathrm{C}$-acetate is due to increased uptake, or simply a consequence of reduced production of AcCoA 
from glucose. In addition, as with glutamine, labelling of lipogenic AcCoA from acetate may not track with net carbon contribution. Such mismatch between labelling and actual carbon contribution could happen if the turnover of protein acetylation occurs at a much higher rate than fatty acid production, leading to exchange as discussed above. Therefore, efforts to more accurately quantify fluxes are required to better understand how hypoxic cells maintain lipogenesis. Similarly, much is still to be learned about acetate production and availability in vivo.

Beyond the changes in AcCoA production, fatty acid maturation has also been found to be affected by hypoxic conditions. Specifically, the desaturase responsible for the production of the abundant fatty acid oleate, SCD, is oxygen-dependent. Our work revealed a decrease in the cellular desaturation index (oleate/ stearate), suggesting reduced SCD activity in hypoxia, which was confirmed by tracing experiments (Kamphorst et al, 2013). As a consequence, hypoxic cells were more reliant on exogenous monounsaturated fatty acids to maintain a viable desaturation index. A separate study corroborated reduced SCD activity and hence increased dependence on exogenous mono-unsaturated fatty acids in hypoxic cancer cells (Young et al, 2013). Cells with unregulated growth exposed to limited oxygen and serum were found to be unable to maintain a viable desaturation index, resulting in induction of unfolded protein response-mediated apoptosis due to sustained endoplasmic reticulum stress.

Our work with a panel of isogenic cell lines harbouring distinct oncogenic alterations, suggests there is variability in the response to SCD inhibition (Kamphorst et al, 2013). Cells with constitutive activation of the $\mathrm{PI}(3) \mathrm{K} / \mathrm{Akt} / \mathrm{mTOR}$ pathway (through myr-Akt expression) had a high fatty acid biosynthetic flux. These cells were extremely sensitive to SCD inhibition due to a strongly decreasing desaturation index and an inability to compensate by scavenging serum lipids. Strikingly, expression of mutant Ras decreased de novo lipogenesis and increased the ability to scavenge serum lipids. As a result, growth of these cells was unaffected by SCD inhibition when provided with sufficient serum. It should be noted, however, that the effects of Ras on de novo lipogenesis and lipid scavenging might be context and cell type dependent (Ricoult et al, 2016). Variability in response is also seen in vivo. Silencing of SCD in prostate orthografts strongly increased the survival of the host mice (Peck et al, 2016). However, xenografted MiaPaCa-2 (PDAC) tumours were entirely refractory against SCD inhibition (Daemen et al, 2015). Thus, although certain cancer cells and tumours rely heavily on de novo lipogenesis (either through specific oncogenic alteration and/or a limited availability in exogenous lipids), this is certainly not universally true in all tumour settings. On the basis of this, interrupting lipid scavenging may prove to be an effective approach in a subset of cancers, and especially in notoriously hypoxic tumours.

What are the most relevant sources of fatty acids for cancer cells, and how are they taken up? Although it is hard to establish the availability of the various substrates in the tumour microenvironment, some guidance can be found in plasma concentrations. The free fatty acids palmitate and oleate, for instance, have mean concentrations of $66-122 \mu \mathrm{M}$ and $51-122 \mu \mathrm{M}$, respectively (Psychogios et al, 2011). Palmitate containing lysophosphatidylcholine (LPC(16:0)), one of the most abundant lysolipids, has a similar mean concentration of $106 \mu \mathrm{M}$, and the oleate containing LPC(18:1) is present at approximately $40 \mu \mathrm{M}$. Much higher concentrations are found in cholesterol esters that are sequestered in lipoproteins. The palmitate cholesterol ester has a reported mean concentration of $405 \mu \mathrm{m}$ and oleate cholesterol $750 \mu \mathrm{M}$. The triglyceride content of LDLs is relatively low $(<10 \mathrm{wt} \%)$ but higher in lower-density particles. Although lysolipid uptake and metabolism remain poorly described, more is known about the mechanisms of intake for free fatty acid and lipoproteins and their involvement in cancer (Figure 2). One of the best
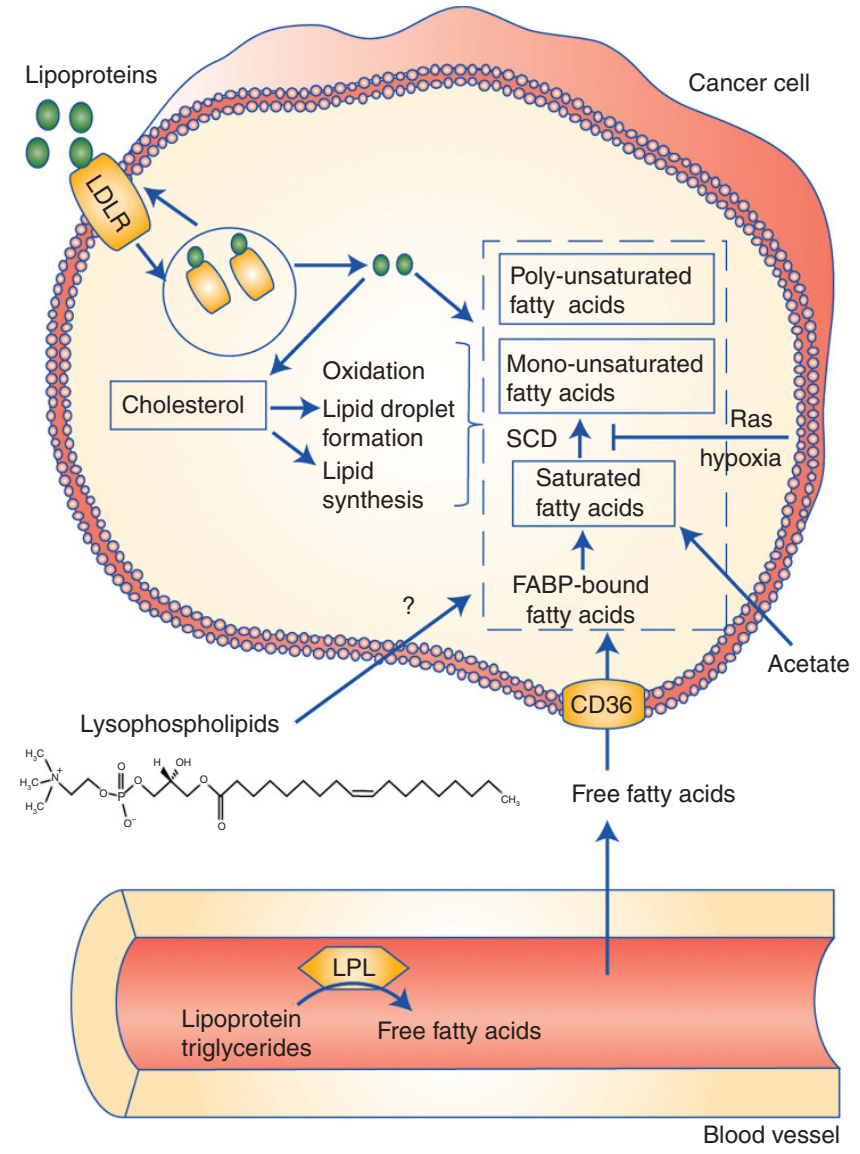

Figure 2. Lipid-acquisition routes in cancer cells. Cancer cells can produce non-essential fatty acids from the primary nutrients glucose and glutamine. However, low nutrient availability and hypoxia compromises the ability to synthesise fatty acids from these substrates and diminishes the ability to desaturate fatty acids by the oxygendependent SCD. The presence of mutant Ras also leads to decreased desaturation by SCD. Cancer cells use acetate as an alternative substrate for fatty acid synthesis. In addition, they acquire fatty acids (particularly unsaturated ones) and cholesterol directly through LDLRmediated uptake and processing. Furthermore, free fatty acids (either generated by lipoprotein lipase (LPL) or circulating free fatty acids) can be transported into the cell via CD36. Lysophospholipids may also be a physiologically relevant source for unsaturated fatty acids, but how they are catabolised remains unknown to date.

characterised fatty acid transporters is the fatty acid translocase CD36, which facilitates transport of circulating free fatty acids across the cell membrane. In addition, fatty acids sequestered in lipoproteins can be released extracellularly by lipoprotein lipase (LPL) followed by CD36-mediated uptake, or lipoproteins can be internalised in their entirety through LDL receptor-mediated endocytosis. Strikingly, one study found that while expression of both LPL and CD36 in cultured cancer cells is generally low, it is widespread in tumour tissues such as breast, liposarcoma and prostate tissues (Kuemmerle et al, 2011). CD36 has additionally been shown to be associated with progression of hepatocellular carcinoma (Nath et al, 2015), and in other cancers LDL receptor (LDLR) is upregulated, including in PDAC tumours (Guillaumond et al, 2015). In PDAC, LDLR is highly expressed both in vitro and in vivo. Consequently, PDAC cells maintain high levels of cholesterol and silencing LDLR reduced cell proliferation. In human PDAC, LDLR expression correlates with poor prognosis. Finally, lipoprotein ingestion via macropinocytosis may also occur. 
One question lingers: what is the availability of exogenous lipids in the tumour microenvironment, especially in hypoxic regions where we know fatty acid maturation is compromised? Just like other cells, cancer cells need essential PUFA, so some supply is absolutely necessary. One could argue that an insufficient perfusion not only leads to a scarcity of primary nutrients and oxygen but also of serum lipids. However, in some tumour types, such as breast cancer, the cancer cells may be in close proximity to adipocytes (Tan et al, 2011). Also, blood vessels in tumours are typically poorly constructed and leaky, and tumours have lymphatic deficiency. As a result, macromolecules and large complexes such as albumin and lipoproteins might actually accumulate in tumours. Detailed in vivo tracing experiments together with systematic histological evaluation will be the way forward in addressing these questions.

\section{SUMMARY}

The discovery that beyond glucose and glutamine cancer cells can metabolise a variety of substrates, including macromolecules, is both bad and good news. Bad news because it demonstrates a degree of metabolic adaptability that likely makes biosynthetic inhibitors have limited impact for certain tumour types. Good news because these scavenging pathways potentially provide new and selective therapeutic opportunities, on their own or in combination with biosynthesis inhibitors. The collective of recent, conceptually innovative papers has established metabolic scavenging as a new paradigm. However, many important questions remain. Does macropinocytosis only occur in pancreatic cancer? How important is it really in supporting metabolism, in vivo? And does it provide a mechanism of resistance to for example mTOR inhibition? And with respect to lipid metabolism, to what degree do various tumours in vivo depend on de novo fatty acid synthesis $v s$ uptake and what are the most important routes of fatty acid acquisition? We anticipate that answers to many of these questions will be found in the coming years, and that the therapeutic potential of scavenging pathways will become clearer.

\section{ACKNOWLEDGEMENTS}

The Kamphorst lab is supported by funding from Cancer Research UK and the Rosetrees Trust. JJK is a Cancer Research UK Career Development Fellow (C50242/A17728). The figures were created using images provided by pubchem and Servier Medical Art (http://www.servier.co.uk/content/servier-medical-art). Owing to space constraints, we apologise for failing to cite many relevant papers.

\section{CONFLICT OF INTEREST}

The authors declare no conflict of interest.

\section{REFERENCES}

Bloomfield G, Traynor D, Sander SP, Veltman DM, Pachebat JA, Kay RR (2015) Neurofibromin controls macropinocytosis and phagocytosis in Dictyostelium. Elife 4: 1-25.

Boone BA, Bahary N, Zureikat AH, Moser AJ, Normolle DP, Wu W-C, Singhi AD, Bao P, Bartlett DL, Liotta LA, Espina V, Loughran P, Lotze MT, Zeh HJ (2015) Safety and biologic response of pre-operative autophagy inhibition in combination with gemcitabine patients with pancreatic adenocarcinoma. Ann Surg Oncol 22: 4402-4410.
Chauhan VP, Boucher Y, Ferrone CR, Roberge S, Martin JD, Stylianopoulos T, Bardeesy N, Depinho RA, Padera TP, Munn LL, Jain RK (2014) Compression of pancreatic tumor blood vessels by hyaluronan is caused by solid stress and not interstitial fluid pressure. Cancer Cell 26: 14-15.

Comerford SA, Huang Z, Du X, Wang Y, Cai L, Witkiewicz AK, Walters H, Tantawy MN, Fu A, Manning HC, Horton JD, Hammer RE, McKnight SL, Tu BP (2014) Acetate dependence of tumors. Cell 159: 1591-1602.

Commisso C, Davidson SM, Soydaner-Azeloglu RG, Parker SJ, Kamphorst JJ, Hackett S, Grabocka E, Nofal M, Drebin JA, Thompson CB, Rabinowitz JD, Metallo CM, Vander Heiden MG, Bar-Sagi D (2013) Macropinocytosis of protein is an amino acid supply route in Ras-transformed cells. Nature 497: 633-637.

Daemen A, Peterson D, Sahu N, McCord R, Du X, Liu B, Kowanetz K, Hong R, Moffat J, Gao M, Boudreau A, Mroue R, Corson L, O’Brien T, Qing J, Sampath D, Merchant M, Yauch R, Manning G, Settleman J, Hatzivassiliou G, Evangelista M (2015) Metabolite profiling stratifies pancreatic ductal adenocarcinomas into subtypes with distinct sensitivities to metabolic inhibitors. Proc Natl Acad Sci USA 112: E4410-E4417.

Deberardinis RJ, Sayed N, Ditsworth D, Thompson CB (2008) Brick by brick: metabolism and tumor cell growth. Curr Opin Genet Dev 18: 54-61.

Delgiorno KE, Carlson MA, Osgood R, Provenzano PP, Brockenbough JS, Thompson CB, Shepard HM, Frost GI, Potter JD, Hingorani SR (2014) Response to Chauhan et al.: interstitial pressure and vascular collapse in pancreas cancer-fluids and solids, measurement and meaning. Cancer Cell 26: $16-17$.

Dumontier M, Höcht P, Mintert U, Faix J (2000) Rac1 GTPases control filopodia formation, cell motility, endocytosis, cytokinesis and development in Dictyostelium. J Cell Sci 113: 2253-2265.

Elstrom RL, Bauer DE, Buzzai M, Karnauskas R, Harris MH, Plas DR, Zhuang H, Cinalli RM, Alavi A, Rudin CM, Thompson CB (2004) Akt stimulates aerobic glycolysis in cancer cells. Cancer Res 64: 3892-3899.

Engelman JA, Luo J, Cantley LC (2006) The evolution of phosphatidylinositol 3-kinases as regulators of growth and metabolism. Nat Rev Genet 7: 606-619.

Fan J, Kamphorst JJ, Rabinowitz JD, Shlomi T (2013) Fatty acid labeling from glutamine in hypoxia can be explained by isotope exchange without net reductive isocitrate dehydrogenase (IDH) flux. J Biol Chem 288: 31363-31369.

Goldstein D, El-Maraghi RH, Hammel P, Heinemann V, Kunzmann V, Sastre J, Scheithauer W, Siena S, Tabernero J, Teixeira L, Tortora G, Van Laethem J-L, Young R, Penenberg DN, Lu B, Romano A, Von Hoff DD (2015) nab-Paclitaxel plus gemcitabine for metastatic pancreatic cancer: longterm survival from a phase III trial. J Natl Cancer Inst 107: 1-10.

Guillaumond F, Bidaut G, Ouaissi M, Servais S, Gouirand V, Olivares O, Lac S, Borge L, Roques J, Gayet O, Pinault M, Guimaraes C, Nigri J, Loncle C, Lavaut MN, Garcia S, Tailleux A, Staels B, Calvo E, Tomasini R, Iovanna JL, Vasseur S (2015) Cholesterol uptake disruption, in association with chemotherapy, is a promising combined metabolic therapy for pancreatic adenocarcinoma. Proc Natl Acad Sci USA 112: 2473-2478.

Hoeller O, Bolourani P, Clark J, Stephens LR, Hawkins PT, Weiner OD, Weeks G, Kay RR (2013) Two distinct functions for PI3-kinases in macropinocytosis. J Cell Sci 126: 4296-4307.

Kamphorst JJ, Chung MK, Fan J, Rabinowitz JD (2014) Quantitative analysis of acetyl-CoA production in hypoxic cancer cells reveals substantial contribution from acetate. Cancer Metab 2: 23.

Kamphorst JJ, Cross JR, Fan J, de Stanchina E, Mathew R, White EP, Thompson CB, Rabinowitz JD (2013) Hypoxic and Ras-transformed cells support growth by scavenging unsaturated fatty acids from lysophospholipids. Proc Natl Acad Sci USA 110: 8882-8887.

Kamphorst JJ, Nofal M, Commisso C, Hackett SR, Lu W, Grabocka E, Vander Heiden MG, Miller G, Drebin JA, Bar-Sagi D, Thompson CB, Rabinowitz JD (2015) Human pancreatic cancer tumors are nutrient poor and tumor cells actively scavenge extracellular protein. Cancer Res 75: 544-553.

Karsli-Uzunbas G, Guo JY, Price S, Teng X, Laddha SV, Khor S, Kalaany NY, Jacks T, Chan CS, Rabinowitz JD, White E (2014) Autophagy is required for glucose homeostasis and lung tumor maintenance. Cancer Discov 4 : 914-927.

Kim J-W, Tchernyshyov I, Semenza GL, Dang CV (2006) HIF-1-mediated expression of pyruvate dehydrogenase kinase: a metabolic switch required for cellular adaptation to hypoxia. Cell Metab 3: 177-185.

Kuemmerle NB, Rysman E, Lombardo PS, Flanagan AJ, Lipe BC, Wells WA, Pettus JR, Froehlich HM, Memoli VA, Morganelli PM, Swinnen JV, Timmerman LA, Chaychi L, Fricano CJ, Eisenberg BL, Coleman WB, Kinlaw WB. Lipoprotein lipase links dietary fat to solid tumor cell proliferation (2011) Mol Cancer Ther 10: 427-436. 
Metallo CM, Gameiro PA, Bell EL, Mattaini KR, Yang J, Hiller K, Jewell CM, Johnson ZR, Irvine DJ, Guarente L, Kelleher JK, Vander Heiden MG, Iliopoulos O, Stephanopoulos G (2012) Reductive glutamine metabolism by IDH1 mediates lipogenesis under hypoxia. Nature 481: 380-384.

Mullen AR, Wheaton WW, Jin ES, Chen PH, Sullivan LB, Cheng T, Yang Y, Linehan WM, Chandel NS, DeBerardinis RJ (2012) Reductive carboxylation supports growth in tumour cells with defective mitochondria. Nature 481: 385-388.

Nath A, Li I, Roberts LR, Chan C (2015) Elevated free fatty acid uptake via CD36 promotes epithelial-mesenchymal transition in hepatocellular carcinoma. Sci Rep 5: 14752.

Palm W, Park Y, Wright K, Pavlova NN, Tuveson DA, Thompson CB (2015) The utilization of extracellular proteins as nutrients is suppressed by mTORC1. Cell 162: 259-270.

Papandreou I, Cairns RA, Fontana L, Lim AL, Denko NC (2006) HIF-1 mediates adaptation to hypoxia by actively downregulating mitochondrial oxygen consumption. Cell Metab 3: 187-197.

Pavlova NN, Thompson CB (2016) The emerging hallmarks of cancer metabolism. Cell Metab 23: 27-47.

Peck B, Schug ZT, Zhang Q, Dankworth B, Jones DT, Smethurst E, Patel R, Mason S, Jiang M, Saunders R, Howell M, Mitter R, Spencer-Dene B, Stamp G, McGarry L, James D, Shanks E, Aboagye EO, Critchlow SE, Leung HY, Harris AL, Wakelam MJ, Gottlieb E, Schulze A (2016) Inhibition of fatty acid desaturation is detrimental to cancer cell survival in metabolically compromised environments. Cancer Metab 4: 6.

Perera RM, Stoykova S, Nicolay BN, Ross KN, Fitamant J, Boukhali M, Lengrand J, Deshpande V, Selig MK, Ferrone CR, Settleman J, Stephanopoulos G, Dyson NJ, Zoncu R, Ramaswamy S, Haas W, Bardeesy N (2015) Transcriptional control of autophagy-lysosome function drives pancreatic cancer metabolism. Nature 524: 361-365.

Porstmann T, Santos CR, Griffiths B, Cully M, Wu M, Leevers S, Griffiths JR, Chung YL, Schulze A (2008) SREBP activity is regulated by mTORC1 and contributes to Akt-dependent cell growth. Cell Metab 8: 224-236.

Provenzano PP, Cuevas C, Chang AE, Goel VK, Von Hoff DD, Hingorani SR (2012) Enzymatic targeting of the stroma ablates physical barriers to treatment of pancreatic ductal adenocarcinoma. Cancer Cell 21: 418-429.

Psychogios N, Hau DD, Peng J, Guo AC, Mandal R, Bouatra S, Sinelnikov I, Krishnamurthy R, Eisner R, Gautam B, Young N, Xia J, Knox C, Dong E, Huang P, Hollander Z, Pedersen TL, Smith SR, Bamforth F, Greiner R, McManus B, Newman JW, Goodfriend T, Wishart DS (2011) The human serum metabolome. PLoS One 6: e16957.
Rabinowitz JD, White E (2010) Autophagy and metabolism. Science 330: 1344-1348.

Ricoult SJ, Yecies JL, Ben-Sahra I, Manning BD (2016) Oncogenic PI3K and K-Ras stimulate de novo lipid synthesis through mTORC1 and SREBP. Oncogene 35: 1250-1260.

Schug ZT, Peck B, Jones DT, Zhang Q, Grosskurth S, Alam IS, Goodwin LM, Smethurst E, Mason S, Blyth K, McGarry L, James D, Shanks E, Kalna G, Saunders RE, Jiang M, Howell M, Lassailly F, Thin MZ, Spencer-Dene B, Stamp G, van den Broek NJ, Mackay G, Bulusu V, Kamphorst JJ, Tardito S, Strachan D, Harris AL, Aboagye EO, Critchlow SE, Wakelam MJ, Schulze A, Gottlieb E (2015) Acetyl-CoA synthetase 2 promotes acetate utilization and maintains cancer cell growth under metabolic stress. Cancer Cell 27: 57-71.

Strohecker AM, White E (2014) Autophagy promotes BrafV600E-driven lung tumorigenesis by preserving mitochondrial metabolism. Autophagy 10: 384-385.

Tan J, Buache E, Chenard MP, Dali-Youcef N, Rio MC (2011) Adipocyte is a non-trivial, dynamic partner of breast cancer cells. Int J Dev Biol 55: 851-859.

Warburg O, Wind F, Negelein E (1927) The metabolism of tumors in the body. J Gen Physiol 8: 519-530.

Warburg O (1956) On the origin of cancer cells. Science 123: 309-314.

Wise DR, Ward PS, Shay JES, Cross JR, Gruber JJ, Sachdeva UM, Platt JM, DeMatteo RG, Simon MC, Thompson CB (2011) Hypoxia promotes isocitrate dehydrogenase-dependent carboxylation of $\alpha$-ketoglutarate to citrate to support cell growth and viability. Proc Natl Acad Sci USA 108: 19611-19616.

Yang S, Wang X, Contino G, Liesa M, Sahin E, Ying H, Bause A, Li Y, Stommel JM, Dell'antonio G, Mautner J, Tonon G, Haigis M, Shirihai OS, Doglioni C, Bardeesy N, Kimmelman AC (2011) Pancreatic cancers require autophagy for tumor growth. Genes Dev 25: 717-729.

Young RM, Ackerman D, Quinn ZL, Mancuso A, Gruber M, Liu L, Giannoukos DN, Bobrovnikova-Marjon E, Diehl JA, Keith B, Simon MC (2013) Dysregulated mTORC1 renders cells critically dependent on desaturated lipids for survival under tumor-like stress. Genes Dev 27: 1115-1131.

(c) (1) (2) This work is licensed under the Creative Commons cc) ${ }_{\mathrm{BY}} \mathrm{NC}$ SA Attribution-Non-Commercial-Share Alike 4.0 International License. To view a copy of this license, visit http:// creativecommons.org/licenses/by-nc-sa/4.0/ 